\title{
Stichwort: Grounding
}

\author{
Von äußerem Grund und innerer Mitte
}

\author{
Ulfried Geuter
}

K aum ein Konzept in der Körperpsychotherapie eint die leibpädagogische und die psychoanalytische Tradition der Körperpsychotherapie so sehr wie das Konzept der Erdung. Seit Alexander Lowens Bioenergetik wird es auch im Deutschen oft als Grounding bezeichnet. In der Leibpädagogik sah schon Hedwig Kallmeyer, die Lehrerin von Elsa Gindler, Entspannung als ein „Abgeben der Schwere" an (Arps-Aubert 2012, 299). Gindler arbeitete in ihren Kursen daran, das Gleichgewicht und die Schwerkraft im Stehen, Sitzen oder Liegen zu erfahren (Arps-Aubert 2012, $277 \mathrm{ff}$ ). Aus ihren Kursnotizen ist das Zitat überliefert: „Die Erde ist notwendig, damit wir unsere Kräfte einsetzen können“ (Ludwig 2002, 133). Zu stehen betrachtete sie als eine Möglichkeit, „im Gleichgewicht zu sein und dadurch zur Ruhe zu kommen“ (Ludwig 2002, 153).

Alexander Lowen führte die Arbeit im Stehen zu einer Zeit in die Psychotherapie ein, als PatientInnen nur liegen oder allenfalls sitzen sollten (Büntig 1983). Lowen (1979) ging davon aus, dass ein Mensch im Stehen das Gefühl erfahren könne, mit einem tragenden Grund verbunden, und das heiße geerdet zu sein. Diese Verbindung zu spüren, hielt er für heilsam. Denn wer gut geerdet sei, könne „nicht gehemmt oder verklemmt sein“ (Lowen 1979, 169). Gut geerdet zu sein, war für ihn daher ein Hauptziel der Therapie.

Ausgehend von diesen beiden Traditionen haben fast alle Schulen der Körperpsychothe- rapie bis hin zur Tanztherapie das Konzept des Groundings aufgegriffen (De Tord / Bräuninger 2015).

Grounding wird in der Körperpsychotherapie wörtlich und metaphorisch verstanden, wie das Wort auch im Englischen verwendet wird. Es meint in einem wörtlichen Sinne, „die Tragfähigkeit des Bodens und des eigenen Stützapparates zu spüren “ (Braunbarth 2009, 107), im übertragenen Sinn die Fähigkeit, einen festen Stand einzunehmen und für sich selbst eintreten zu können (Westland 2015, 190). Erdung kann man als eine körperliche Prüfung der Verbindung zur äußeren Realität bezeichnen (Ehrensperger 2006). Im Stehen können wir uns vorstellen, im Boden verwurzelt zu sein wie ein Baum. Stehen gilt daher im Sensory Awareness als ein Antidot zur Angst. Erdung lässt sich insoweit als Coping-Technik zur Bewältigung überwältigender oder heftiger Gefühle nutzen. In der Akutbehandlung von posttraumatischer Übererregung ist sie ein Mittel, die in der Dissoziation verlorengegangene Verbindung zur Wirklichkeit wieder zu bahnen und sich im Hier und Jetzt zu reorientieren. Erdend wirkt dabei nicht nur das Stehen, sondern jede körperliche Erfahrung, in der wir den Kontakt zum tragenden Grund spüren, beispielsweise auch im Angelehnt-Sein an eine Wand. Belz-Knöferl und Brown (2006) unterscheiden ein vertikales Erden im Stehen von einem horizontalen Erden im Liegen. 
Boadella (1991) spricht davon, dass wir uns im „äußeren Grund“ erden und im „inneren Grund“ (S.108) zentrieren. Sich in sicherem Stehen, Sitzen oder Liegen mit der äußeren Wirklichkeit und dem tragenden Grund zu verbinden, ergänzen wir in der Körperpsychotherapie durch das Zentrieren (Geuter 2019, $229 \mathrm{ff}$ ). Zentrieren bedeutet, sich in seinem inneren Schwerpunkt, in der inneren und zugleich physischen Mitte des Unterbauchs, dem Zentrum der körperlichen Schwerkraft zu sammeln (Schmid-Bergmann 2011). Aus der inneren Mitte und aus dem Überlassen an den äußeren Grund heraus zu leben, sind gleichermaßen Fähigkeiten der Selbstregulation (Aposhyan 2004, $131 \mathrm{ff}$ ).

Erdung kann man nicht nur als eine Erfahrung in der Beziehung zur materiellen Wirklichkeit verstehen, sondern auch als eine in der Beziehung zu anderen Menschen. Der erste Boden, auf dem das kleine Kind ruht und sich später gehalten an seinen Händen mit den Füßen aufrichtet, ist nämlich der Körper der Mutter oder des Vaters (Moser 2001, 108). Auch später werden wir durch unsere Beziehungen im Leben getragen. Rolef Ben-Shahar $(2014,170)$ spricht dementsprechend von einem ,intersubjektiven Grounding“ in menschlichen Beziehungen. Auch verweist er auf eine „spirituelle Erdung“ im Sinne einer Verbindung mit einer höheren Kraft (ähnlich: Ehrensperger 2006).

Die Art, wie ein Mensch körperlich steht, kann in diagnostischer Hinsicht Hinweise darauf geben, wie er im Leben steht (Röhricht 2000, 94): fest und beweglich oder starr und unsicher, auf etwas zugehend oder zurückweichend, eingesackt oder aufgerichtet, überstreckt oder gerade. Ehrensperger (1996) empfiehlt das Stehen auf einer Balancierscheibe, um damit verbundene Spannungsmuster zu diagnostizieren. Den Stand zu verändern, kann Spannungsmuster verändern und einen Patienten eher in einen hedonischen Tonus angenehmer und entspannter Kernaffektivität brin- gen (Geuter 2015, 189 ff). Das kann aber auch dazu beitragen, fehlende Spannung aufzubauen oder wieder ins Lot zu kommen, wenn der Körper in der Hypotonie aus der Achse gefallen ist.

In den verschiedenen Traditionen der Körperpsychotherapie wurden dazu unterschiedliche Methoden und Techniken entwickelt. Einige Beispiele:

- einen sicheren Stand einnehmen, bei dem das Gewicht gleichmäßig auf die Füße verteilt ist, der körperliche Schwerpunkt im Becken gespürt und die Wirbelsäule aufgerichtet wird;

- in aufrechter Bewegung gehen, wobei der Kopf aus der Wirbelsäule herausgestreckt wird, ohne ihn nach hinten zu überstrecken; Hausmann und Neddermeyer $(1996,207)$ sprechen davon, den Kopf aus den Schultern wachsen zu lassen;

- im Rhythmus der Atmung im Stehen das Becken nach hinten und nach vorne kippen;

- mit dem Einatmen leicht in die Knie und mit dem Ausatmen nach oben gehen, ohne die Knie dabei durchzudrücken (Sollmann 1988).

Downing $(1996,85)$ plädiert dafür, bei solchen Übungen nicht dynamisch zu arbeiten, sondern in langsamen und ruhigen kleinen Veränderungen eine genaue Wahrnehmung zu fördern. Genaue Wahrnehmung erleichtert den Transfer von der Wahrnehmung des körperlichen Stands zu einer möglichen symbolischen Bedeutung. Dabei sollten wir nicht von einer Eins-zu-eins-Beziehung zwischen den körperlichen Phänomenen und ihrer Bedeutung ausgehen, sondern die konkrete subjektive Bedeutung genau erkunden (Geuter 2015, 280 f).

Eine bekannte Übung zum Grounding ist der sogenannte bioenergetische Bogen. Bei dieser Übung stehen die Füße schulterbreit, man setzt die Fäuste auf den Beckenkamm, spannt die Ellenbogen am Rücken aufeinander zu und schiebt mit gebeugten Knien das Becken nach vorne, so dass der Oberkörper über die Fäuste 
und Arme nach hinten kommt und der Druck auf die Fußballen zunimmt, ohne dass sich die Füße vom Boden lösen (Lowen / Lowen 1988). Diese Übung zielt nicht auf eine genaue Wahrnehmung, sondern auf eine dynamische Veränderung des Spannungszustands, bei der die Muskeln so unter einen willkürlich verstärkten Zug gesetzt werden, dass sie festgehaltene Spannungen freigeben, oft durch ein Zittern in den Beinen oder in der Bauch- und Beckenmuskulatur. In der bioenergetischen Therapie wird diese Übung zuweilen mehrfach wiederholt (Koemeda-Lutz / Steinmann 2004). Auftretende Spannungen werden über eine Übung gelöst, die Der Elefant genannt wird. Hier beugt man sich nach vorne und lässt das Gewicht des Kopfes und der Arme Richtung Boden sinken, um danach langsam wieder in der Wirbelsäule von unten nach oben hochzukommen (Geuter 2019, 240 f). Ein weiteres Ziel dieser Übung ist es, durch den dynamischen Wechsel zwischen intensivierter Spannung und Entspannung das Vitalniveau anzuheben.

Wichtig bei allen Erdungsübungen ist es, die Aufmerksamkeit immer auch auf den Atemfluss zu richten. Ein gehaltener Atem steht der Verbindung zum tragenden Grund im Wege. In seine eigene Mitte, in die Verbindung mit dem inneren Grund kann man nur kommen, wenn die Atmung und die mit ihr verbundene Atembewegung den ganzen Körper durchströmen können und wenn es einen ungehinderten Fluss im Rhythmus zwischen Ein- und Ausatmen gibt. Eine solche Zentrierung schafft „Gleichgewicht, Offenheit, Erdung und innere Ruhe“ (Blackburn / Price 2007, 70).

Erdung ohne innere Aufmerksamkeit für das, was körperlich und seelisch geschieht, kann hilfreich sein. Psychotherapeutisch wirksam wird sie nur, wenn der / die PatientIn in innerer Aufmerksamkeit dabei ist. Nur dann können bestehende Muster erfahren werden, der erste Schritt zu ihrer Transformation.

\section{Literatur}

Aposhyan, S. (2004): Body-mind psychotherapy. Principles, techniques, and practical application. Norton, New York

Arps-Aubert, E. von (2012): Das Arbeitskonzept von Elsa Gindler (1885-1961) dargestellt im Rahmen der Gymnastik der Reformpädagogik. Dr. Kovač, Hamburg

Belz-Knöferl, A., Brown, M. (2006): Horizontales Grounding. In: Marlock, G., Weiss, H. (Hrsg.): Handbuch der Körperpsychotherapie. Schattauer, Stuttgart, 699-708

Blackburn, J., Price, C. (2007): Implications of presence in manual therapy. Journal of Bodywork and Movement Therapies 11, 68-77

Boadella, D. (1991): Befreite Lebensenergie. Einführung in die Biosynthese. Kösel, München

Braunbarth, I. (2009): Angststörungen. In: Waibel, M. J., Jakob-Krieger, C. (Hrsg.): Integrative Bewegungstherapie. Störungsspezifische und ressourcenorientierte Praxis. Schattauer, Stuttgart, 99-121

Büntig, W. (1983): Bioenergetik. In: Corsini, R. J. (Hrsg.): Handbuch der Psychotherapie. Beltz, Weinheim, 66-110

De Tord, P., Bräuninger, I. (2015): Grounding: Theoretical application and practice in dance movement therapy. The Arts in Psychotherapy 43, 16-22

Downing, G. (1996): Körper und Wort in der Psychotherapie. Kösel, München

Ehrensperger, T. (2006): Erdung in der therapeutischen Arbeit und im Alltag. In: Marlock, G., Weiss, H. (Hrsg.): Handbuch der Körperpsychotherapie. Schattauer, Stuttgart, 692-698

Ehrensperger, T. (1996): Erdung in der therapeutischen Arbeit und im Alltag. In: Ehrensperger, T. (Hrsg.): Zwischen Himmel und Erde. Beiträge zum Grounding-Konzept. Schwabe \& Co., Basel, 75-89

Geuter, U. (2019): Praxis Körperpsychotherapie. 10 Prinzipien der Arbeit im therapeutischen Prozess. Berlin, Springer

Geuter, U. (2015): Körperpsychotherapie. Grundriss einer Theorie für die klinische Praxis. Berlin, Springer

Hausmann, B., Neddermeyer, R. (1996): Bewegt Sein. Integrative Bewegungs- und Leibtherapie in der Praxis. Junfermann, Paderborn 
Koemeda-Lutz, M., Steinmann, H. (2004): Implikationen neurobiologischer Forschungsergebnisse für die Körperpsychotherapie unter spezieller Berücksichtigung der Affekte. Psychotherapie Forum 12, 88-97

Lowen, A. (1979): Bioenergetik. Therapie der Seele durch Arbeit mit dem Körper. Rowohlt, Reinbek

Lowen, A., Lowen, L. (1988): Bioenergetik für jeden. Das vollständige Übungshandbuch. Peter Kirchheim, München

Ludwig, S. (2002): Elsa Gindler - von ihrem Leben und Wirken. „Wahrnehmen, was wir empfinden“. Hans Christians, Hamburg

Moser, T. (2001): Berührung auf der Couch. Formen der analytischen Körperpsychotherapie. Suhrkamp, Frankfurt

Röhricht, F. (2000): Körperorientierte Psychotherapie psychischer Störungen. Hogrefe, Göttingen

Rolef Ben-Shahar, A. (2014): Touching the relational edge. Body psychotherapy. Karnac, London
Schmid-Bergmann, I. (2011): Hara - die Erdmitte des Menschen. Theorie des Erdens im Taiji Quan. Energie \& Charakter 42 (35), 79-90

Sollmann, U. (1988): Bioenergetik in der Praxis. Rowohlt, Reinbek

Westland, G. (2015): Verbal and non-verbal communication in psychotherapy. Norton, New York

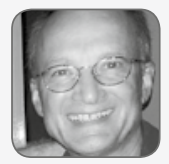

Prof. Dr. Ulfried Geuter

Psychotherapeut in Berlin, a. pl. Prof. im Masterstudiengang Motologie der Universität Marburg, Studienschwerpunkt Körperpsychotherapie. Lehrtherapeut, Lehranalytiker und Dozent in der psychotherapeutischen Aus- und Weiterbildung.

$\equiv$ Prof. Dr. Ulfried Geuter Otto-von-Wollank-Str. 57 | D-14089 Berlin u.geuter@gmx.de 